\title{
Spindle Cell Sarcoma of Thyroid: A Case Report
}

\author{
A. K. Abdul Siyad • Praveen Gopi • N. P. Jayan • \\ Joy Augustine
}

Received: 1 May 2014 / Accepted: 17 September 2014 /Published online: 8 October 2014

(C) Indian Association of Surgical Oncology 2014

\begin{abstract}
Spindle cell sarcoma of thyroid represents a rare entity that may arise primarily from thyroid gland as a variant of thyroid cancer or as metastatic. We report a case of 60 year old man who presented with 2 months history of rapidly growing thyroid enlargement with no obstructive features and preliminary diagnosis of spindle cell lesion of thyroid was made with cytological examination. He underwent total thyroidectomy and histological examination revealed spindle cell sarcoma of thyroid confirmed by immunohistochemical reactions. He was subjected to adjuvant chemoradiotherapy and is on follow up. Sarcoma of thyroid is rarely encountered in routine practice and is diagnosed by microscopic evaluation and immunohistochemical staining which determines the cellular origin and histologic type of tumor. Sarcoma thyroid is best managed with surgery and adjuvant chemoradiotherapy according to the grade of the lesion. In addition overall prognosis in thyroid sarcoma is poor due to late presentation and aggressive nature of the tumour.
\end{abstract}

Keywords Sarcoma Thyroid

\author{
A. K. A. Siyad $\cdot$ P. Gopi $(\bowtie) \cdot$ N. P. Jayan \\ Department of Surgery, Government Medical College, Thrissur, \\ kerala, India \\ e-mail: drpg111@yahoo.co.in \\ A. K. A. Siyad \\ e-mail: abdulsiyadak@gmail.com \\ N. P. Jayan \\ e-mail: Jayan.np.7@gmail.com \\ J. Augustine \\ Department of Pathology, Government Medical College, Thrissur, \\ kerala, India \\ e-mail: joyajoy@rediffmail.com
}

\section{Introduction}

Spindle cell Sarcomas of the thyroid gland are rare entities and are not encountered routinely in clinical practice or in the context of thyroid pathology. It is described as a malignant neoplasm of mesenchymal origin composed of elongated and spindle shaped cells. They can be primary or metastatic in origin. Spindle cell lesions may originate from reactive or neoplastic process. They mainly grow denovo, but can also arise in a postscald cicatrix, site of previous trauma, post radiotherapy [1].

We report a rare case of spindle cell sarcoma in a male patient presenting with an anterior neck swelling.

\section{Case Presentation}

A 60 year old man was admitted in our surgery ward with rapidly growing mass in the midline of neck for 2 months. He had no previous medical problems. He was a chronic alcoholic and smoker. Physical examination revealed a non tender,firm nodular, $3 \times 3 \mathrm{~cm}$ mass in the right lobe of thyroid, moving with deglutition and had no enlarged cervical lymph nodes. His thyroid profile was in normal range. USG neck showed a large lobulated hypoechoic nodule in the right lobe of thyroid with calcifications. The cytological evaluation revealed follicular epithelial cells and spindle cells in clusters suggestive of a spindle cell lesion (Fig. 1). Further imaging with MRI neck demonstrated a well difined exophytic lesion in right lobe of thyroid with moderate heterogenous enhancement which abuts the right sternocleidomastoid with no infiltration to other structures and no enlarged cervical lymph nodes. Chest xray was normal. With the preliminary diagnosis of spindle cell lesion of thyroid, we planned surgical exploration. 


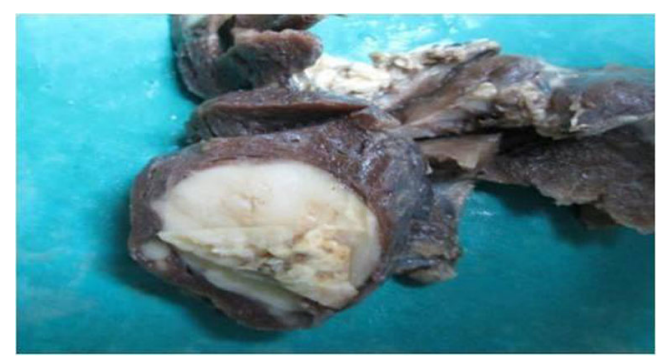

Fig. 1 FNAC showing spindle cells

Intraoperatively, a $3 \times 2 \mathrm{~cm}$ hard nodule was identified on right lobe of thyroid with no fixity or enlarged cervical lymph nodes and we proceeded with total thyroidectomy. Post op period was uneventful. On gross examination of the specimen a $3 \times 2.5 \times 2.5 \mathrm{~cm}$ circumscribed grey white nodule with calcified area in right lobe and a normal left lobe was noted (Fig. 2). Microscopiclly, all margins were free of neoplasm. Histologically, Spindle cells in fascicles with scanty-moderate eosinophilic cytoplasm, elongated plump vesicular nuclei and mild pleomorphism some showing nucleoli and sprinkled lymphocytes in between was observed (Fig. 3) with evidence of capsular invasion (Fig. 4) and mitosis (1-2/HPF). Further immunohistochemical staining, (Fig. 5) showed positivity with vimentin (a) and negative results with Thyroglobulin (b), Cytokeratin (c), Calcitonin (d), BCL-2 (e), CD 5 (f), CD 34 (g), CD 68 (h), S-100 (i). The final diagnosis of spindle cell sarcoma thyroid, intermediate grade was made. Patient was referred to our Radiotherapy department where he had his adjuvant chemotherapy of 6 cycles with Ifosamide $(2 \mathrm{~g})$ \& Doxorubicin $(70 \mathrm{mg})$ per cycle followed by external beam radiotherapy of $45 \mathrm{~Gy}$ and a booster dose of $15 \mathrm{~Gy}$. Currently, patient is on Thyroxine 100ug and under follow up for 2 years with no evidence of recurrence or metastasis.

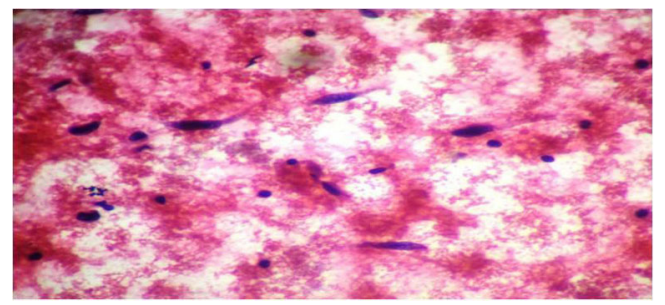

Fig. 2 Gross cut section specimen showing well circumscribed area

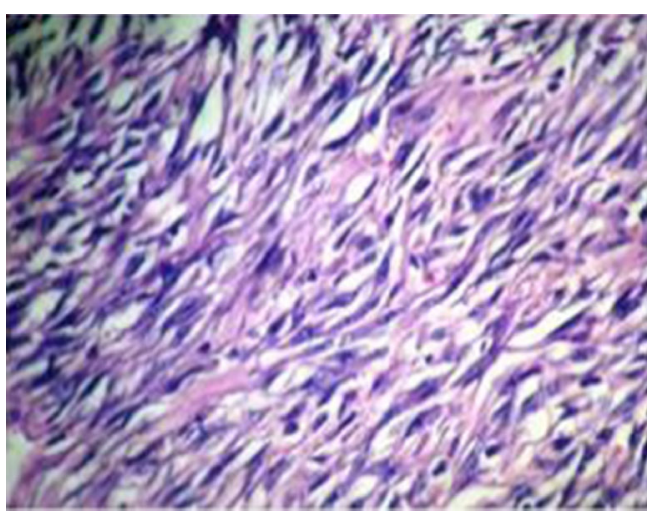

Fig. 3 Microscopic section showing spindle cells in clusters

\section{Discussion}

Sarcomas of the thyroid are rare and comprise less than $1 \%$ of all the thyroid malignancies. Only limited description of this condition is present in the literature. We report this case report due to its rarity and diagnostic challenge. $15-20 \%$ of what occur in head and neck and $0.014 \%$ of sarcoma are primary of the thyroid [2]. Primary lesions include leiomyosarcoma, liposarcoma,fibrosarcoma, malignant fibrous histiocytoma (MFH), Malignant peripheral nerve sheath tumor (MPNT). Differential diagnosis of spindle cell lesions include anaplastic carcinoma, medullary carcinoma, Reidel's thyroiditis, intrathyroidal thymoma, metaplastic spindle cell proliferation associated with follicular cell derived tumors, teratoma of the thyroid, synovial sarcoma, Post fine needle aspiration spindle cell nodules of the thyroid (PSCNT), Spindle epithelial tumor with thymus like differentiation (SETTLE), Carcinoma showing thymus like differentiation (CASTLE) [3].

Sarcomas present in elderly patients with long standing history of goitre. Cases have been described in those with

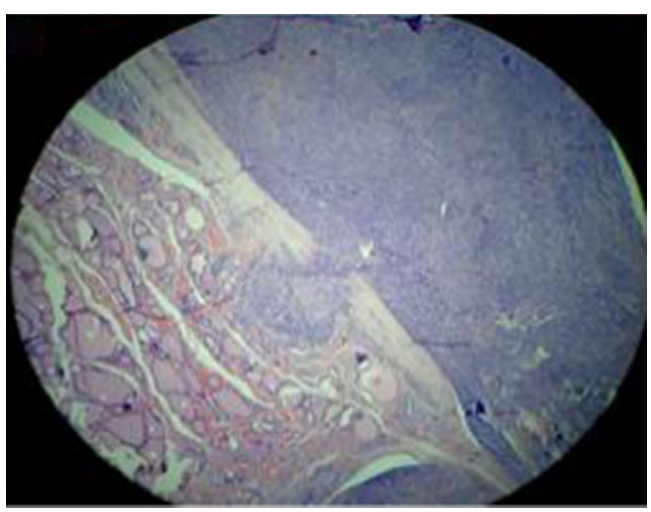

Fig. 4 Microscopic section showing capsular invasion 


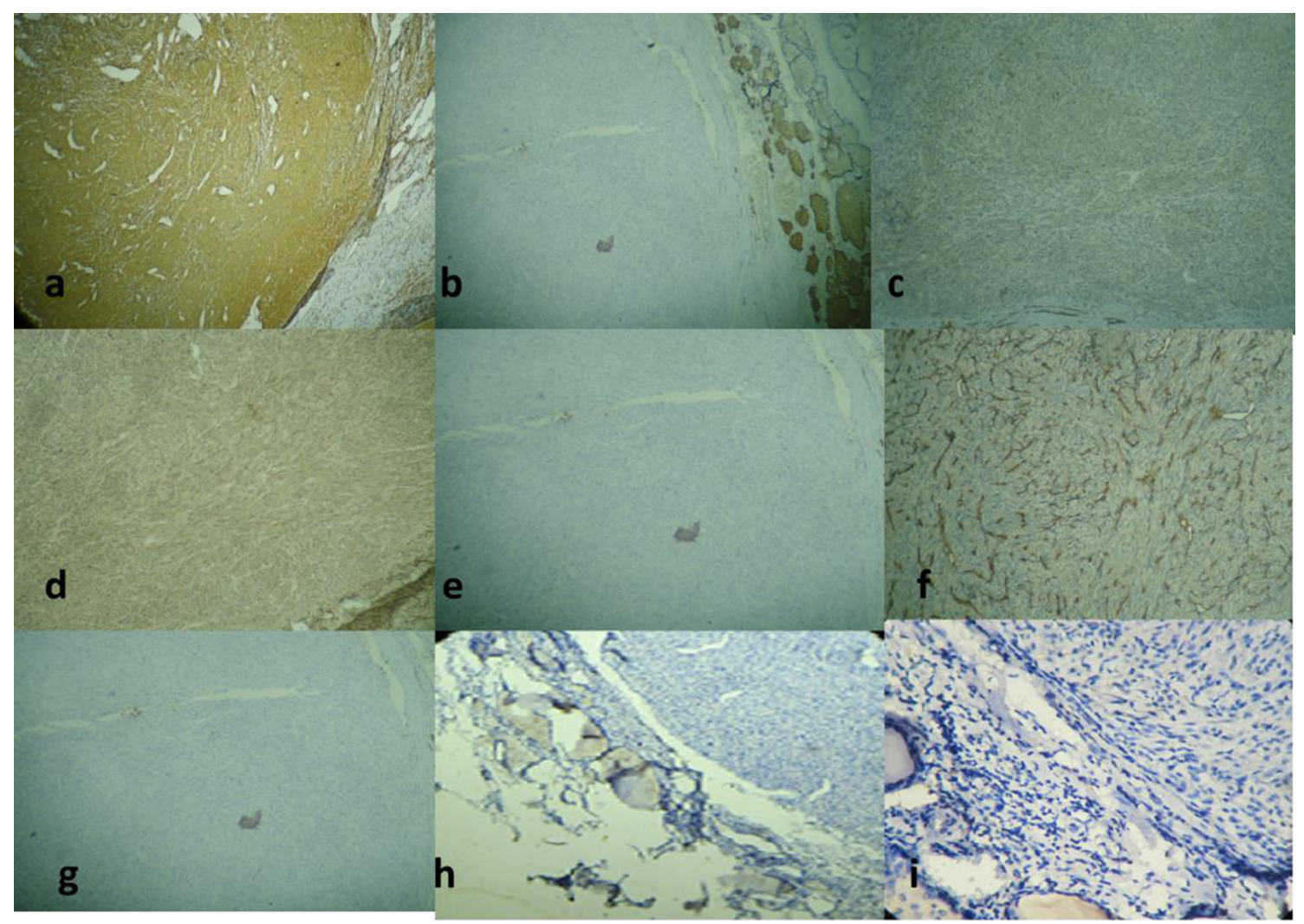

Fig. 5 Immunohistochemical stains. a) Vimentin b) Thyroglobulin c) Cytokeratin d) Calcitonin e) BCL-2 f) CD34 g) CD5 h) CD68 i) S 100

prior history of external beam radiation therapy. Clinically there is a mass in the thyroid gland with or without local invasion and lymph node metastasis. At the time of presentation, half of patients have distant metastasis and common sites of involvement are the lungs, bones and brain [4]. For staging, imaging of neck with CT, MRI is required. Diagnosis is confirmed with the cytology, histopathology and immunohistochemistry is required for appropriate classification.

Management of sarcomas of thyroid is surgical resection with total thyroidectomy and removal of any involved lymph nodes or adjacent structures. Tumours with advanced pathological features (extracapsular spread, high mitotic rate, nuclear pleomorphism, unresectable disease) warrant postoperative radiotherapy and chemotherapy [4]. Prognosis depends upon the histological grade.
Conflicts of Interest No potential conflict of interest relevant to this article was reported.

\section{References}

1. Sami Titi, Kinga Sycz, Marek Uminski (2007) Primary Fibrosarcoma of the Thyroid Gland - a case report. Poland Journal of Pathology 58(1):59-62

2. Vianna D, Otavio Curioni, Luciano Franca et al. (2012) The histological rarity of Thyroid cancer. Brazilian Journal of Otorhinolaryngology 78(4):48-51. http://www.bjorl.org

3. Papi G, Corrado S, Virginia A (2006) LiVolsi. Primary Spindle Cell Lesion of Thyroid gland. An Overview. Am J Clin Pathol 125 Suppl: S95-S123

4. Sniezek JC, Holtel M (2003) Rare tumors of the Thyroid gland. Otolaryngol Clin North Am 36(1):107-15 